\title{
BMJ Open Quality Evaluation of a patient safety programme on Surgical Safety Checklist Compliance: a prospective longitudinal study
}

\section{Brigid M Gillespie, ${ }^{1,2,3}$ Emma L Harbeck, ${ }^{3}$ Joanne Lavin, ${ }^{1}$ Kyra Hamilton, ${ }^{4}$ Therese Gardiner, ${ }^{5}$ Teresa K Withers, ${ }^{6}$ Andrea P Marshall ${ }^{1,2,3}$}

To cite: Gillespie BM, Harbeck EL, Lavin J, et al. Evaluation of a patient safety programme on Surgical Safety Checklist Compliance: a prospective longitudinal study.BMJ Open Quality 2018;7:e000362. doi:10.1136/ bmjoq-2018-000362

- Additional material is published online only. To view please visit the journal online (http://dx.doi.org/10.1136/ bmjoq-2018-000362).

Received 17 February 2018 Revised 2 June 2018 Accepted 14 June 2018

\section{Check for updates}

(c) Author(s) (or their employer(s)) 2018. Re-use permitted under CC BY-NC. No commercial re-use. See rights and permissions. Published by BMJ.

For numbered affiliations see end of article.

Correspondence to Dr Brigid M Gillespie; b.gillespie@griffith.edu.au

\section{ABSTRACT}

Background Surgical Safety Checklists (SSC) have been implemented widely across 132 countries since 2008. Yet, despite associated reductions in postoperative complications and death rates, implementation of checklists in surgery remains a challenge. The aim of this study was to assess the impact of a patient safety programme over time on SSC use and incidence of clinical errors.

Design A prospective longitudinal design over three time points and a retrospective secondary analysis of clinical incident data was undertaken.

Methods We implemented a patient safety programme over 4 weeks to improve surgical teams' use of the SSC. We undertook structured observations to assess surgical teams' checklist use before and after programme implementation and conducted a retrospective audit of clinical incident data 12 months before and 12 months following implementation of the programme.

Results There were significant improvements in the observed use of the SSC across all phases, particularly in sign-out where completion rates ranged from $79.3 \%$ to 94.5\% ( $p<0.0001)$ following programme implementation. Across clinical incident audit periods, 33019 surgical procedures were performed. Based on a subsample of 64 cases, clinical incidents occurred in 22/16 $264(0.13 \%)$ before implementation and 42/16 755 (0.25\%) cases after implementation. The most predominant incident after programme implementation was inadequate tissue specimen labelling (23/42, 54.8\%). Clinical incidents resulted in minimal or no harm to the patient.

Conclusions The benefit in using a surgical checklist lies in the potential to enhance team communications and the promotion of a team culture in which safety is the priority.

\section{INTRODUCTION}

Estimates indicate that over 234 million patients undergo surgical procedures worldwide every year. ${ }^{1}$ Adverse events in surgery were reported to occur in up to $14 \%$ of patients, ${ }^{2}$ while mortality rates were reported to be between $1 \%$ and $4 \% .{ }^{34}$ In an effort to improve morbidity and mortality rates associated with surgery, the WHO Surgical Safety Checklist (SSC) was developed in 2008. In the decade since its introduction, implementation of the WHO SSC has become increasingly widespread and it is now used in over
132 countries worldwide. ${ }^{5}$ Many studies, ${ }^{6-10}$ including several meta-analyses, ${ }^{11-13}$ have reported reductions in associated postoperative complications since the introduction of the SSC. In more proximal outcomes, Haynes et $a t^{6}$ reported improvements resulting in increases in correct surgical site marking from $56 \%$ to $83 \%$, and general improvements in safety processes from $34 \%$ to $57 \%$.

\section{Problem description}

Despite that checklist use has become more widespread, results have not been so impressive and have been mixed or inconclusive. ${ }^{1415}$ Some studies have shown that although $100 \%$ compliance with the SSC was documented, in reality, checklists were completed less than $10 \%$ of the time when operating room (OR) staff were observed. ${ }^{16}$ Checklists may contribute to improved patient safety outcomes; however, they are often implemented as part of multicomponent quality improvement initatives. ${ }^{14}$ Additionally, there is mounting evidence identifying contextual and organisational challenges in relation to ease of checklist adoption into workflow patterns, ${ }^{17} 18$ and their true impact on patient safety. ${ }^{16} 1920$ These aspects require high commitment to practice change at all levels, particularly relative to physician involvement and organisational support. ${ }^{21}$ Unsurprisingly, many healthcare organisations achieve only partial implementation success when they are initiated. ${ }^{22} 23$

To date, few studies have evaluated the observed use of the SSC longitudinally following the implementation of a patient safety programme. The objectives of this study were to: (1) assess checklist item completeness rates before and after implementation of a patient safety programme designed to improve surgical teams' participation in safety checks using the SSC; and (2) describe the impact of the intervention on the prevalence of clinical errors. 


\section{METHODS}

\section{Study overview}

We conducted a prospective longitudinal study using structured observations and undertook a retrospective secondary analysis of electronic database audit. The primary outcomes included observed SSC participation and completion rates, while the secondary outcome was period prevalence of clinical errors. Following baseline measures, we implemented a patient safety programme over 4 weeks and collected observational measures at 3 and 12 months' postimplementation. We undertook the audit of electronic secondary outcome data retrospectively, 12 months before and 12 months after programme implementation. Online supplementary file 1 details the data collection time points, method of collection and measures across implementation phases.

\section{Context}

The study setting was a 750-bed university hospital in Queensland, Australia. The facility had 16 commissioned operating rooms, catering for all specialties except transplantation, and performs approximately 16000 surgeries each year. Surgical teams consisted of anaesthetists and surgeons and their registrars, and nurses working in circulating/instrument and anaesthetic roles. As the programme was implemented department-wide, all staff $(\mathrm{n}=150)$ from surgery, anaesthetics and nursing were invited to participate in the preimplementation and postimplementation using structured observations. The clinical incident data were drawn from a consecutive sample of patients who underwent elective, emergent and emergency surgeries at the study site over a 24-month period between October 2014-2015 and November 2015-2016. The Pass The Baton (PTB) programme was delivered in November 2015.

\section{Intervention}

The ultimate goal of the WHO SSC is to ensure that surgical teams consistently follow several critical safety steps, thus minimise the most common and avoidable risks endangering the well-being of patients. ${ }^{1}$ The original 19-item three SSC checkpoints include: sign-in (preinduction), time-out (surgical pause prior to commencement of the procedure) and sign-out (checks performed during or just after skin closure). The WHO recommends that a designated coordinator (often the circulating nurse) review verbally each checkpoint with the appropriate team member(s) to confirm that all key actions for that phase of care have been performed. ${ }^{1}$ Having a dedicated person lead the checklist process ensures that safety steps are not missed or rushed in an effort to progress with the next phase of the procedure. However, while this person is often the circulating nurse, any member of the surgical team can coordinate the checklist. ${ }^{1}$

At the study hospital, the WHO SSC was adapted to meet the needs of the local context and included 20 items across the sign-in, time-out and sign-out checkpoints. Although mandated throughout the OR department, the SSC was haphazardly used and inconsistently completed, with varying levels of staff participation. ${ }^{24}$ The patient safety programme, $P T B$, was implemented department-wide, across all surgical specialties to increase item coverage and team participation across the three checkpoints. Development of the PTB programme was informed by a series of studies ${ }^{151824}$ conducted at the study site. The most significant barriers to using the SSC as recommended were workflow, limited knowledge about timing/ content of checks, a lack of clinical leadership and dissonant attitudes. ${ }^{184}$

Prior to implementing the $P T B$ programme, two team members (BMG and JL) had initial discussions to identify other interested staff members. BMG has had a longstanding association as a researcher in the OR department, and is well known to many of the nursing and medical staff. JL is a senior nurse, working as a perioperative educator. In this role, she has implemented other types of practice improvement initiatives. As a staff member, JL is highly regarded, and considered an opinion leader. JL identified a mix of nurses working across all perioperative areas to champion PTB programme. Both BMG and JL approached several physicians in surgery and anaesthetics to garner support. The implementation team consisted of anaesthetic/circulating/instrument nurses, a senior physician and two researchers. The clinicians on the team were experts in their respective fields and were highly respected influential leaders within the department. The programme was coproduced with staff across surgery and anaesthetics. A logic model (ie, a diagram describing structures in place to deliver the programme, the intended activities and intended short, medium and long-term outcomes) ${ }^{25}$ was developed a priori by the implementation team. The PTB programme, delivered over 4 weeks, contained process strategies designed to facilitate behaviour change, including change champions, audit and feedback, education, and prompts and reminders. ${ }^{21}$ To address the issues identified with workflow, ${ }^{18}$ the $P T B$ intervention itself was nurse led, and involved the allocation of the anaesthetic nurse to pass on case-relevant information obtained at the sign-in checkpoint (in the induction room) to other surgical team members who were in the OR, at an appropriate time. The operating surgeon led the time-out checkpoint and the circulating nurse was the designated to lead sign-out. The process to execute the time-out and sign-out checkpoints involved the deliberate use of a scripted checklist in a 'call and response' process, adequate engagement by the OR team, appropriate timing to consider workflow, coverage and duration for all items of the checklist.

\section{Measures}

Structured observations

We evaluated checklist participation/completion using behaviour descriptors based on previous literature. ${ }^{26-28}$ The data collectors, both experienced OR nurses, received study-specific training in observational methods. The three-part structured observational tool had 70 
items, including 50 items having a 'yes'/'no' response to indicate items covered, team members who were present and/or initiated each checkpoint, whether there was active participation, time/location of checks and free text to document field notes. Prior to data collection, the observational tool was pilot tested and inter-rater reliability was established. During pilot testing of the tool in the field, observers further clarified and cross-checked ratings for items, and consensus achieved. For each procedure, we tallied the number of team members who were present, initiated and participated in the execution of the SSC. We timed each checkpoint in minutes/seconds and measured length of surgery in minutes. Observers also documented field notes to give context around the interactions, actions and behaviours of team members.

\section{Clinical incident data}

In this study, we defined a 'clinical error' as any error in judgement or conduct in the clinical setting, regardless of whether or not the error leads to or causes an adverse event. ${ }^{29}{ }^{30}$ We specifically focused on clinical errors such as surgical injuries, wrong site surgery, mistaken patient identities, mislabelling of human tissue specimens and retained foreign adjuncts/objects. The Severity Assessment Code (SAC) classification system ${ }^{31}$ was used in coding of clinical incidents/adverse events (online supplementary appendix) Characteristics of clinical incidents were coded based on cause, type, reason/issue, study phase and patient outcome (ie, none, minimal, medium, high level of harm) in an Excel database.

\section{Data collection}

We collected observational and clinical incident data over 27 months, spanning October 2014-2015 (preimplementation) through to November 2015-2016 (postimplementation). Observers recorded whether all items in sign-in, time-out and sign-out were attempted. Observers recorded three measures of quality: whether all specified information was communicated, whether all members were present and whether there was active participation. We also collected case-related information relative to team membership, personnel present, length of surgery and specialty. For the retrospective clinical incident audit, the database administrator drew data from the clinical incident information database. The lead author received these deidentified data in an encrypted Excel database. Participating and reporting of clinical incidents in the system is compulsory, and all public hospitals in Queensland have an electronic local risk management system. Once the system has been populated with incident data, the reports are linked to a state-wide repository. Local hospitals around Queensland submit incident data directly to the repository that collates, cleans, stores and uses it for the purposes of analysis. Online supplementary file 1 tabulates time points, methods of data collection and measures for data collected prospectively and retrospectively over the study period.

\section{Analysis}

Observational and clinical incident data were analysed using the Statistical Package for Social Sciences (SPSS; V.23, IBM). We cleaned the data and checked a random sample of $20 \%$ for accuracy. Descriptive analyses included absolute (n) and relative frequencies (\%) to analyse categorical variables (role, qualifications, surgical specialty, checklist item completion, clinical error type), while means/SD were used for continuous data (numbers participated in checklist and number of items used). We calculated the mean compliance rate for each section of the checklist based on the sum of the number of items in each section divided by the total number of items (ie, behaviours/actions actually observed) in the checklist. We used inferential analyses using t-tests to examine mean differences across time points relative to the WHO SSC completion rates (sign-in, time-out, sign-out). Inter-rater reliability was calculated using interclass correlation coefficient (ICC) for observational data. A $p$ value of $<0.05$ was considered significant and $95 \%$ CIs were used.

\section{Ethics}

Ethics approval was given by the ethics committees of the hospital (HREC/13/QGC/154) and the university (NRS/06/14/HREC). Participants gave written consent prior to observations. We advised participants of their rights to discontinue participation anytime during the data collection period. Following ethics approval, we sought permission to obtain deidentified clinical incident data from the Director-General, Queensland Health, as required by the Public Health Act (2005).

\section{RESULTS}

\section{Structured observations}

Table 1 presents descriptive results of team and case-related characteristics across the three time points. In total, 77 surgical teams including anaesthetic and surgical consultants and registrars, residents and nurses were observed over the study period using the WHO SSC.

Table 2 displays descriptive results specifically in relation to checklist initiation and participation, and the proportion of item completion across its three checkpoints. The majority of teams observed across all phases of the study had five to six core ${ }^{\mathrm{i}}$ members ${ }^{32}$ present for each case. Across study time points, checklist compliance ranged from $75.9 \%$ to $82.8 \%$, respectively. Significant improvements were noted in item completion rates across each checkpoint, particularly, in relation to sign-out where completion rates ranged from $79.3 \%$ to $94.5 \%$ ( $\mathrm{p}<0.0001$ ) following implementation of the PTB programme. Inter-rater reliability between observers across phases was adequate (ICC 0.65 , $\mathrm{p}<0.001)$.

\section{Clinical incident data}

Table 3 presents the results of the clinical incident audit. During the preaudit and postaudit periods, 33019

${ }^{\mathrm{i}}$ Core membership is defined as team members who had worked together, weekly or fortnightly, for a minimum of 3 months. ${ }^{32}$ 
Table 1 Descriptive summary for case-related results of observed cases

\begin{tabular}{|c|c|c|c|}
\hline & $\begin{array}{l}\text { Baseline } \\
\text { n (\%) }\end{array}$ & $\begin{array}{l}6 \text { months } \\
\mathrm{n}(\%)\end{array}$ & $\begin{array}{l}12 \text { months } \\
\mathrm{n}(\%)\end{array}$ \\
\hline Number of cases & 10 & 35 & 32 \\
\hline \multicolumn{4}{|l|}{ Core members present } \\
\hline 0-7 (small) & $9(90.0)$ & $31(88.6)$ & $26(81.3)$ \\
\hline 8-11 (medium) & $0(0.0)$ & $4(11.4)$ & $6(18.7)$ \\
\hline$\geq 12$ (large) & $1(10.0)$ & $0(0.0)$ & $0(0.0)$ \\
\hline \multicolumn{4}{|l|}{ Staff assigned to OR } \\
\hline Anaesthetist consultant & $10(100.0)$ & $32(91.4)$ & $18(56.3)$ \\
\hline Anaesthetist registrar & $8(80.0)$ & $23(65.7)$ & $10(31.3)$ \\
\hline Anaesthetic RMO & $2(20.0)$ & $8(22.9)$ & $1(3.1)$ \\
\hline Surgeon consultant & $7(70.0)$ & $26(74.3)$ & $20(62.5)$ \\
\hline Surgeon registrar & $10(10.0)$ & $33(94.3)$ & $22(68.8)$ \\
\hline Surgical RMO & $6(60.0)$ & $6(17.1)$ & $9(28.1)$ \\
\hline Anaesthetist nurse & $10(100.0)$ & $35(100)$ & $32(100)$ \\
\hline Scrub nurse & $10(100.0)$ & $35(100)$ & $23(71.9)$ \\
\hline Scout nurse & $10(100.0)$ & $35(100)$ & $32(100)$ \\
\hline Scout nurse 2 & $9(90.0)$ & $22(62.9)$ & $12(37.5)$ \\
\hline Theatre assistant & $10(100.0)$ & $30(85.7)$ & $16(50.0)$ \\
\hline Other* & $9(90.0)$ & $16(45.7)$ & $11(34.4)$ \\
\hline \multicolumn{4}{|l|}{ Specialty } \\
\hline Cardiac surgical & $0(0.0)$ & $2(5.7)$ & $0(0.0)$ \\
\hline ENT and maxillary facial & $0(0.0)$ & $3(8.6)$ & $3(9.4)$ \\
\hline Eye surgery & $0(0.0)$ & $2(5.7)$ & $7(21.9)$ \\
\hline Gynaecology and obstetrics & $4(40.0)$ & $6(17.1)$ & $3(9.4)$ \\
\hline General & $3(30.0)$ & $3(8.6)$ & $1(3.1)$ \\
\hline Neurosurgical & $0(0.0)$ & $2(5.7)$ & $0(0.0)$ \\
\hline Orthopaedic & $0(0.0)$ & $7(20.0)$ & $3(9.4)$ \\
\hline Paediatrics & $0(0.0)$ & $5(14.3)$ & $0(0.0)$ \\
\hline Urology & $1(10.0)$ & $3(8.6)$ & $14(43.8)$ \\
\hline Vascular & $2(20.0)$ & $2(5.7)$ & $1(3.1)$ \\
\hline \multicolumn{4}{|l|}{ Location of sign-in } \\
\hline Induction room & $10(100)$ & $29(82.9)$ & $27(84.4)$ \\
\hline Operating room & $0(0.0)$ & $3(8.6)$ & $4(12.5)$ \\
\hline Holding bay & $0(0.0)$ & $3(8.6)$ & $1(3.1)$ \\
\hline \multicolumn{4}{|l|}{ Time-out } \\
\hline Before induction & $0(0.0)$ & $1(2.9)$ & $2(6.3)$ \\
\hline During induction & $0(0.0)$ & $1(2.9)$ & $2(6.3)$ \\
\hline Prior knife to skin & $10(100.0)$ & $33(94.3)$ & $28(87.5)$ \\
\hline After knife to skin & $0(0.0)$ & $0(0.0)$ & $0(0.0)$ \\
\hline \multicolumn{4}{|l|}{ Sign-out } \\
\hline Before patient leaves OR & $0(0.0)$ & $30(85.7)$ & 31 (96.9) \\
\hline
\end{tabular}

*Included medical students, midwives, company representative and radiology personnel.

ENT, ear, nose and throat; OR, operating room; RMO, resident medical officer.

surgical procedures were performed, with an increase of 491 cases in the post-test period. A total of 75 incidents among 72 patients were sampled consecutively across the 2-year audit period (ie, at baseline there were 20 patients who had clinical incidents but some had more than one so there were 22 incidents). After 
Table 2 WHO checklist observation results

\begin{tabular}{lllll}
\hline & Baseline & $\mathbf{6}$ months & 12 months & P values* \\
\hline Number of cases & 10 & 35 & 32 & $00: 40(00: 48)$ \\
\hline $\begin{array}{l}\text { Average length of surgery (hour:min) } \\
\quad \text { Range }\end{array}$ & $01: 22(00: 50)$ & $00: 58(00: 58)$ & $00: 05-03: 29$ \\
Core members present & $00: 17-02: 25$ & $00: 04-03: 47$ & & \\
$\quad$ Mean (SD) & & & $6.3(1.8)$
\end{tabular}

Sign-in

Number present

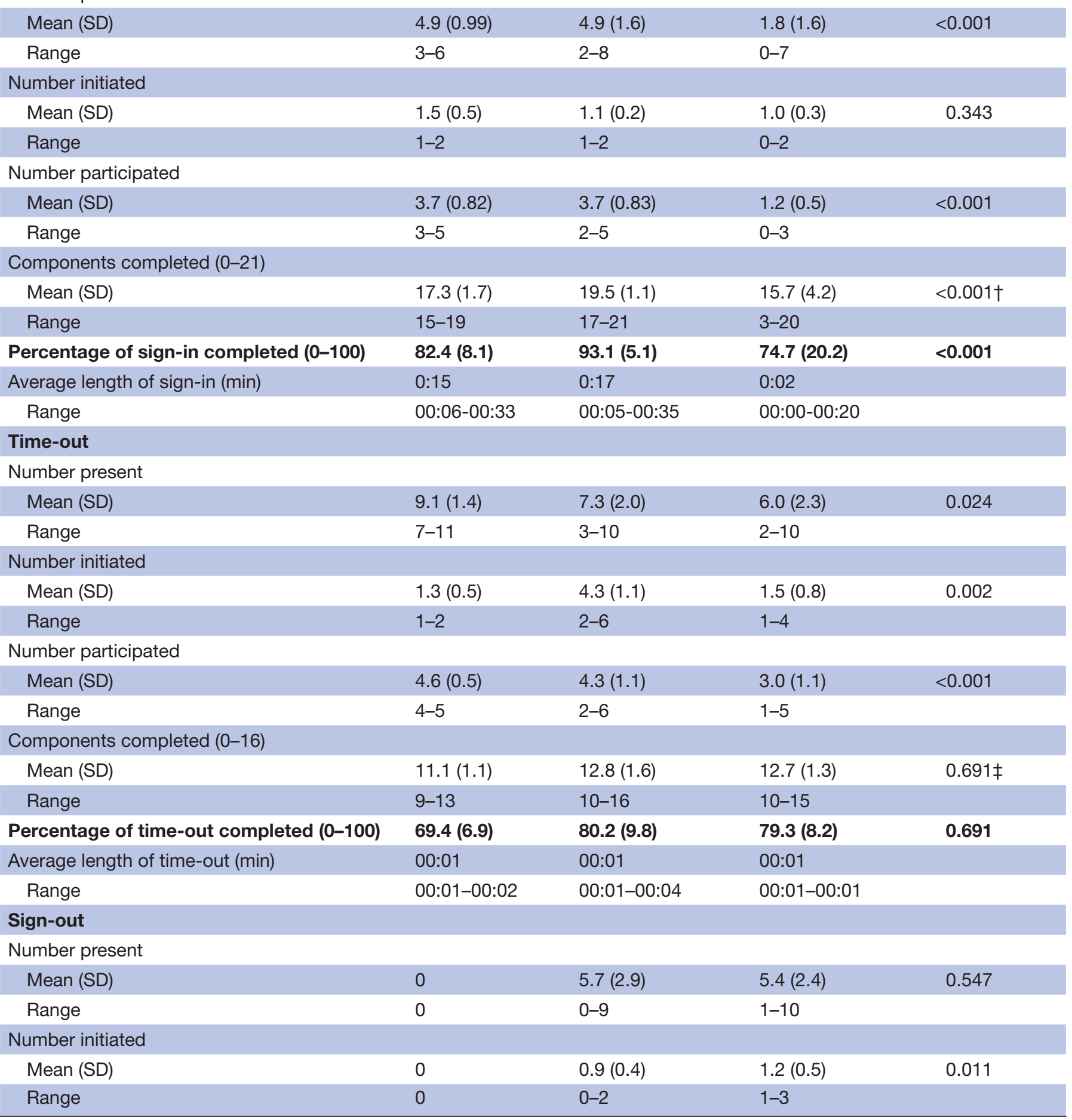


Table 2 Continued

\begin{tabular}{lllll}
\hline & Baseline & $\mathbf{6 m o n t h s}$ & $\mathbf{1 2 \text { months }}$ & P values* $^{*}$ \\
\hline Number participated & & & & \\
$\quad$ Mean (SD) & 0 & $2.9(1.6)$ & $2.5(1.1)$ & 0.261 \\
$\quad$ Range & 0 & $0-6$ & $1-5$ & \\
Components completed (0-12) & 0 & $9.5(3.7)$ & $11.3(1.0)$ & $0.007 \S$ \\
$\quad$ Mean (SD) & 0 & $1-12$ & $8-12$ & \\
$\quad$ Range & 0 & $\mathbf{7 9 . 3 ( 3 0 . 7 )}$ & $\mathbf{9 4 . 5 ( 8 . 1 )}$ & $\mathbf{0 . 0 0 7}$ \\
Percentage of sign-out completed (0-100) & $00: 00$ & $00: 00$ & $00: 00$ & \\
Average length of sign-out (min) & $00: 00-00: 00$ & $00: 00-00: 02$ & $00: 00-00: 02$ & \\
$\quad$ Range & &
\end{tabular}

Independent t-test for compliance. Significant $\mathrm{p}$ values in bold text.

${ }^{*} \mathrm{P}$ values represent independent t-tests, comparing baseline 6 months after implementation and 12 months after implementation mean differences.

†Sign-in $t=5.0$ (65), $\mathrm{p}<0.001$, mean difference $=3.9(0.7), 95 \% \mathrm{Cl} 2.4$ to 5.3 .

$\ddagger$ Time-out $t=0.4$ (65), $\mathrm{p}=0.691$, mean difference $=0.1(0.4), 95 \% \mathrm{Cl}-0.6$ to -0.8 .

$\S$ Sign-out $t=-2.7(65), \mathrm{p}=0.007$, mean difference $=-1.8(0.7), 95 \% \mathrm{Cl}-3.2$ to -0.5 .

\begin{tabular}{|c|c|c|c|}
\hline & $\begin{array}{l}\text { Baseline } \\
\text { n (\%) }\end{array}$ & $\begin{array}{l}\text { Postintervention } \\
\text { n (\%) }\end{array}$ & $\chi^{2}$ ( $P$ values) \\
\hline Number of hospital cases & 16264 & 16755 & \\
\hline \multicolumn{4}{|l|}{ Incidence } \\
\hline SAC 3 events* $^{*}$ & $22(100.0)$ & $42(100.0)$ & - \\
\hline \multicolumn{4}{|l|}{ Incident type } \\
\hline Pathology & $7(31.8)$ & $23(54.8)$ & $3.1(0.081)$ \\
\hline Invasive/non-invasive care & $15(68.2)$ & $19(45.2)$ & \\
\hline \multicolumn{4}{|l|}{ Stage of procedure } \\
\hline Specimen collection & $5(22.7)$ & $16(38.1)$ & $3.1(0.377)$ \\
\hline During intervention & $12(54.5)$ & $14(33.3)$ & \\
\hline After intervention & $3(13.6)$ & $5(11.9)$ & \\
\hline Request & $2(9.1)$ & $7(16.7)$ & \\
\hline \multicolumn{4}{|l|}{ Issues } \\
\hline Inadequate/no Labelling & $7(31.8)$ & $23(54.8)$ & $6.0(0.248)$ \\
\hline Incorrect count & $11(50.0)$ & $15(35.7)$ & \\
\hline Retained object/instrument & $3(13.6)$ & $1(2.4)$ & \\
\hline Wrong body part/side/site & $1(4.5)$ & $3(7.1)$ & \\
\hline \multicolumn{4}{|l|}{ Patient outcome } \\
\hline No harm & $20(90.9)$ & $37(88.1)$ & $0.1(0.732)$ \\
\hline Minimal harm & $2(9.1)$ & $5(11.9)$ & \\
\hline
\end{tabular}

*SAC event, Severity Assessment Code. SAC 3 results in minimal or no harm, which is reasonably expected as an outcome of healthcare.

implementation, 40 patients had 42 incidents, again some having more than one incident. However, only 64 incidents among 60 patients were included in this analysis as some were recorded during implementation of the PTB programme and 12 months after implementation (November 2016). We performed a descriptive analysis on the subsample of 64 cases, 22/16 $264(0.13 \%)$ before implementation and $42(42 / 16755(0.25 \%))$ after implementation (table 3), indicating a marginal increase (although non-significant) in the postimplementation period. The number $\mathrm{SAC}^{\mathrm{ii}} 3$ clinical incidents rose by 20 following implementation and the most

${ }^{\text {ii }} S A C=$ Severity Assessment Code is a rating system used to classify and prioritise an incident. Ratings from 1 to 3 are based on the severity of the outcome for the patient. See online supplementary appendix. 
predominant incident was inadequate tissue specimen labelling (23/42, 54.8\%). The majority (20/22, $90.9 \%$ and $37 / 42,88.1 \%$ respectively) of incidents across both phases did not result in any harm.

\section{DISCUSSION}

We believe that the observed improvements in SSC use following implementation of the PTB programme were due to several factors that commonly lead to successful implementation of any process-based practice. These include having a comprehensive stakeholder-driven programme, using a staged stepwise process providing clear communication and real-time feedback, engaging active leadership, and process simplification and modification. ${ }^{1533}$ Our results showed improvements in checklist participation and item usage rates, following the implementation of the $P T B$ programme. $P T B$ was intended to simplify the checking process through addressing behavioural deficits and contextual factors identified during observations, described in earlier work. ${ }^{18} 24$ In other research, the most common reasons health professionals identified for non-compliance were forgetfulness in using the SSC or in addressing some of its elements, ${ }^{172}$ or a lack of time to complete it. ${ }^{151733}$ We observed substantial improvements in the use of sign-out-improvements that were sustained 12 months after implementation of the programme. Prior to implementation of the programme, we did not observe sign-out during baseline observations, thus any improvement in performance from baseline would be significant. Vogts $e t a l^{4}$ suggest that sign-out use is often low because this section is not explicitly linked to a specific event in patient management, unlike sign-in and time-out-which may have catastrophic consequences if these checkpoints are not completed. Sign-out may reduce the likelihood of the most common 'never event', a retained foreign object during surgery. ${ }^{1}$

In our study, inadequate tissue specimen labelling was the most common clinical incident-the ramifications of which can potentially have devastating effects for patients. Labelling errors can result in inappropriate therapy or withholding treatment in patients with unrecognised malignancies. ${ }^{35}$ Observed improvements in use of the checklist did not result in concomitant reductions in clinical incidents. There are three possible explanations for this finding. The first relates to the possibility that incidents may have been under-reported. The second reason centres on the observed increase in the reporting of SAC 3 incidents following implementation of the $P T B$ programme. This increase may be the result of simultaneous improvements in safety culture over time. The third explanation concerns the reach of the $P T B$ programme, which was not as widespread as we would have liked. During the study period, staff changes and turnover occurred, and likely impacted programme spread across all surgical specialties and teams. The impact of team and staff factors and the stability of the workforce may also contribute to the dilution of capacity, that is, skills and attitudes. ${ }^{36}$
Despite a decrease in observed item compliance in the sign-in phase after implementation, our results attest to the overall sustainability of the evidence-informed PTB programme. Clearly, a variety of factors may create conditions that enable initial implementation, their presence or influence often diminishes over time. ${ }^{36}$ Unintentional 'slippage' can occur because of contextual factors such as a lack of resources, staffing conditions or competing demands. $^{37}$ We attribute sustainability of the PTB programme to factors related to both outer (policies) and inner (ie, culture, structure), the programme itself (fit, adaptability), implementation processes (ie, fidelity monitoring, efforts to align the intervention and the setting) and the capacity to sustain (ie, interpersonal processes such as leadership, support). Beyond implementation of $P T B$, the clinical leads on the implementation team used fidelity maintenance strategies such as audit and feedback, reminders, and provided real-time informal education and training support. The PTB programme was co-constructed with stakeholders, ${ }^{21}$ and 'normalised' in everyday practice over time. ${ }^{38}$ The implementation team included credible leaders from nursing and surgery, which contributed to its sustainability and spread. Following evaluation of the $P T B$ programme at this facility, the programme was implemented at two smaller satellite hospitals within the health services district. Spread of the $P T B$ programme to these facilities was augmented by the leadership of senior nursing staff who work across these hospitals. While implementation fidelity is important, modification and evolution of any programme and intervention may need to occur in response to shifting priorities and contextual influences, or in light of new evidence. ${ }^{36}$ Nonetheless, the importance of having an ongoing education programme cannot be understated given the continuing challenges brought about by staffing changes and attrition.

\section{Limitations}

Few studies have used prospective approaches to evaluate the implementation of practice change interventions. ${ }^{39}$ This is one of the first longitudinal studies to prospectively and retrospectively evaluate a patient safety programme designed to improve surgical teams' participation in, and use of, the SSC. Nonetheless, we acknowledge some limitations. First, the use of a single hospital site and sampling methods of prospective data collection may limit generalisability and increase selection bias. Second, we assessed surgical teams' use of the SSC with direct observations, with results showing moderate consistency between raters, likely due to some variation in interpretation of events. To minimise this, we developed a data dictionary, and the two observers were experienced OR nurses trained in observational research methods. Third, the observational nature of the study may have given rise to the Hawthorne effect. Yet it is likely that this diminished over time as participants became accustomed to the presence of the observers. Fourth, at the time of the implementation, the $P T B$ programme was not mandated, therefore, participation was essentially voluntary, potentially 
leading to self-selection. However, since its introduction, the PTB programme has been included in the health services policy, and has been subsequently introduced at two similar hospital sites across the health services district. Fifth, clinical incident data are secondary, self-reported and represent a non-random sample of errors from an unknown universe of errors. As such, these data cannot generate incidence rates or capture the entire universe (ie, denominator) of errors. Finally, there may have been some misclassification in the coding of harm and other variables. Despite these limitations, our study has considerable strengths in terms of its longitudinal nature and use of data triangulation. These results offer unique insights into SSC use and the types of clinical incidents identified in surgery. We anticipate these results will drive further improvements in checklist implementation and use beyond the facility where the PTB programme was developed and implemented.

\section{CONCLUSION}

Our study showed improvements in team participation and item adherence. Yet, there were no reductions in clinical incidents after implementation of the $P T B$ programme. Despite this, our results suggest an increase in the reporting of clinical incidents, particularly during the sign-out period (ie, specimens, surgical count) increased after implementation. Adaptive changes in the department culture brought about through the introduction of targeted programmes such as $P T B$, although taking longer to achieve, may have a greater impact and lead to more sustainable improvements than simply concentrating on the technical aspects of the checklist. Clearly, the benefit in using a surgical checklist lies in the potential to enhance team communications and the promotion of a team culture in which safety is a high priority.

\section{Author affiliations}

${ }^{1}$ School of Nursing and Midwifery, Griffith University, Gold Coast, Queensland, Australia

${ }^{2}$ Nursing \& Midwifery Research \& Education Unit, Gold Coast Hospital and Health Service, Gold Coast, Queensland, Australia

${ }^{3}$ National Centre of Research Excellence in Nursing, Griffith University, Gold Coast, Queensland, Australia

${ }^{4}$ School of Applied Psychology, Griffith University, Mt Gravatt Campus, Mount Gravatt, Queensland, Australia

${ }^{5}$ Nursing and Midwifery Education and Research Unit, Gold Coast Hospital and Health Service, Gold Coast, Queensland, Australia

${ }^{6}$ Surgical and Procedural Services, Gold Coast Hospital and Health Service, Gold Coast, Queensland, Australia

Contributors BMG planned the study and led all aspects of the project. ELH analysed the data. APM, ELH, KH and TKW provided input in relation to methodology and subject matter. BMG, TG, JL and TKW collected the data. BMG drafted the manuscript. All authors participated in interpretation of the study results, assisted in manuscript revision and approved the final version of the manuscript.

Funding BMG was supported by a National Health and Medical Research Council (NHMRC) (Australia) Translation into Practice (TRIP) Fellowship and the Australian College of Perioperative Nurses (ACORN) Research Grant.

Competing interests None declared.

Patient consent Not required.
Ethics approval Ethics approval was given by the ethics committees of the hospital (HREC/13/QGC/154) and the university (NRS/06/14/HREC).

Provenance and peer review Not commissioned; externally peer reviewed.

Data sharing statement The data contributing to these analyses are held on a secure database held by the lead author.

Open access This is an open access article distributed in accordance with the Creative Commons Attribution Non Commercial (CC BY-NC 4.0) license, which permits others to distribute, remix, adapt, build upon this work non-commercially, and license their derivative works on different terms, provided the original work is properly cited, appropriate credit is given, any changes made indicated, and the use is non-commercial. See:Ohttp://creativecommons.org/licenses/by-nc/4.0/.

\section{REFERENCES}

1. WHO. Implementation of the Surgical Safety Checklist. Geneva: World Health Organisation, 2008:1-28.

2. Anderson O, Davis R, Hanna GB, et al. Surgical adverse events: a systematic review. Am J Surg 2013;206:253-62.

3. Weiser TG, Regenbogen SE, Thompson KD, et al. An estimation of the global volume of surgery: a modelling strategy based on available data. Lancet 2008;372:139-44.

4. Pearse RM, Moreno RP, Bauer P, et al. Mortality after surgery in Europe: a 7 day cohort study. Lancet 2012;380:1059-65.

5. WHO. Surgical Safety Web map. Geneva: World Health Organization, 2013. (accessed 13 Sep 2014).

6. Haynes AB, Weiser TG, Berry WR, et al. A surgical safety checklist to reduce morbidity and mortality in a global population. N Engl J Med 2009;360:491-9.

7. Haugen AS, Søfteland E, Eide GE, et al. Impact of the World Health Organization's Surgical Safety Checklist on safety culture in the operating theatre: a controlled intervention study. $\mathrm{Br} J$ Anaesth 2013;110:807-15.

8. Askarian M, Kouchak F, Palenik CJ. Effect of surgical safety checklists on postoperative morbidity and mortality rates, Shiraz, Faghihy Hospital, a 1-year study. Qual Manag Health Care 2011;20:293-7.

9. Bliss LA, Ross-Richardson CB, Sanzari LJ, et al. Thirty-day outcomes support implementation of a surgical safety checklist. $J$ Am Coll Surg 2012;215:766-76.

10. van Klei WA, Hoff RG, van Aarnhem EE, et al. Effects of the introduction of the WHO "Surgical Safety Checklist" on in-hospital mortality: a cohort study. Ann Surg 2012;255:44-9.

11. Bergs J, Hellings J, Cleemput I, et al. Systematic review and metaanalysis of the effect of the World Health Organization surgical safety checklist on postoperative complications. Br J Surg 2014;101:150-8.

12. Gillespie B, Chaboyer W, Thalib L, et al. Effect of Using a Safety Checklist in Surgery on Patient Complications: A Systematic Review and Meta-Analysis. Anaesthesiology 2014;120:1380-9.

13. Borchard A, Schwappach DL, Barbir A, et al. A systematic review of the effectiveness, compliance, and critical factors for implementation of safety checklists in surgery. Ann Surg 2012;256:925-33.

14. Ko HC, Turner TJ, Finnigan MA. Systematic review of safety checklists for use by medical care teams in acute hospital settings-limited evidence of effectiveness. BMC Health Serv Res 2011;11:211.

15. Gillespie BM, Marshall A. Implementation of safety checklists in surgery: a realist synthesis of evidence. Implement Sci 2015;10:137.

16. Urbach DR, Govindarajan A, Saskin R, et al. Introduction of surgical safety checklists in Ontario, Canada. N Engl J Med 2014;370:1029-38.

17. Bergs J, Lambrechts F, Simons $\mathrm{P}$, et al. Barriers and facilators related to the evidence a systematic review of the qualitative implementation of surgical safety checklists. BMJ Qual Saf 2015.

18. Gillespie BM, Marshall AP, Gardiner T, et al. Impact of workflow on the use of the Surgical Safety Checklist: a qualitative study. ANZ J Surg 2016;86:864-7.

19. Bosk C, Dixon-Woods M, Pronovost PJ. The art of medicine Reality check for checklists. New Eng J Med 2009;374:444-5.

20. Vats A, Vincent CA, Nagpal K, et al. Practical challenges of introducing WHO surgical checklist: UK pilot experience. BMJ 2010;340:b5433.

21. Gillespie BM, Hamilton K, Ball D, et al. Unlocking the "black box" of practice improvement strategies to implement surgical safety checklists: a process evaluation. $J$ Multidiscip Healthc 2017;10:157-66.

22. Weiner BJ, Amick H, Lee SY. Conceptualization and measurement of organizational readiness for change: a review of the literature 
in health services research and other fields. Med Care Res Rev 2008;65:379-436.

23. Shea CM, Jacobs SR, Esserman DA, et al. Organizational readiness for implementing change: a psychometric assessment of a new measure. Implement Sci 2014;9:15.

24. Gillespie BM, Withers TK, Lavin J, et al. Factors that drive team participation in surgical safety checks: a prospective study. Patient Saf Surg 2016;10:3.

25. Moore G, Audrey S, Barker M, et al. Process evaluation of complex interventions UK Medical Research Council (MRC) guidance. 132. Southhampton, UK: MRC Population Health Sciences Research Network, 2012

26. Fourcade A, Blache JL, Grenier C, et al. Barriers to staff adoption of a surgical safety checklist. BMJ Qual Saf 2012;21:191-7.

27. Gillespie B, Harbeck H EK. Effect of a brief team training program on surgical teams' non-technical skills: An interrupted time-series study. Journal of Patient Safety 2017;7.

28. Morgan L, Hadi M, Pickering S, et al. The effect of teamwork training on team performance and clinical outcome in elective orthopaedic surgery: a controlled interrupted time series study. BMJ Open 2015;5:e006216.

29. Oyebode F. Clinical errors and medical negligence. Med Princ Pract 2013;22:323-33.

30. Kohn L, Corrigan J, Donaldson M. To err is human: Building a safer health system. Washington: National Academy Press, 2000.

31. IOM. Patient safety: achieving a new standard for care. Washington, DC: The National Academies Press, 2004.
32. Gillespie BM, Chaboyer W, Fairweather N. Factors that influence the expected length of operation: results of a prospective study. BMJ Qual Saf 2012;21:3-12.

33. Russ S, Sevdalis N, Moorthy K, et al. A Qualitative evaluation of the barriers and facilitators toward implementation of the WHO Surgical safety checklist across hospitals in england lessons from the "surgical checklist implementation project". An Surg 2014;11:1-

34. Vogts N, Hannam JA, Merry AF, et al. Compliance and quality in administration of a Surgical Safety Checklist in a tertiary New Zealand hospital. N Z Med J 2011;124:48-58.

35. Layfield LJ, Anderson GM. Specimen labeling errors in surgical pathology: an 18-month experience. Am J Clin Pathol 2010;134:466-70.

36. Wiltsey Stirman S, Kimberly J, Cook N, et al. The sustainability of new programs and innovations: a review of the empirical literature and recommendations for future research. Implementation Science 2012;7:17.

37. Bowman CC, Sobo EJ, Asch SM, et al. Measuring persistence of implementation: QUERI Series. Implement Sci 2008;3:21.

38. Gillespie BM, Harbeck E, Lavin J, et al. Using normalisation process theory to evaluate the implementation of a complex intervention to embed the surgical safety checklist. BMC Health Serv Res 2018; $18: 170$

39. Wiltsey Stirman S, Kimberly J, Cook N, et al. The sustainability of new programs and innovations: a review of the empirical literature and recommendations for future research. Implement Sci 2012;7:17. 